\title{
Theory of Frustrated Total Reflection Involving Metallic Surfaces
}

\author{
T. R. Young and B. D. Rothrock
}

(November 30, 1962)

\begin{abstract}
The theory for frustrated total reflection has been developed for the case where the third medium is metallic of complex index. Using parallel polarized light a unique minimum in reflectance occurs at a definite film thickness. Experimental verification of the theory is made and indicates the theory applicable to the precise measurement of thin contact films existing between metallic and dielectric surfaces.
\end{abstract}

\section{Introduction}

In the field of precise length measurement there exists the need to measure precisely the proximity of two surfaces. One or both surfaces may be plane. Frequently at least one of the surfaces is transparent to visible light. For instance, in the interferometric determinations of the lengths of metallic end standards called gage blocks, a transparent plate may be contacted to one of the end surfaces and the optical length between the surface of this plate and the noncontacted surface of the gage block is determined. The practical length, or calibrated length, of the gage block is obtained from the optical length by correcting for the phase change difference occurring at reflection from the two dissimilar surfaces. Thus the practical length of the gage block is dependent upon the thickness of the film existing between the contacted surfaces. A transparent contacting plate, usually of quartz, is often used rather than a plate of material similar to the metallic gage block to facilitate visual examination of the contacted surfaces. A satisfactory contacting is considered to be one that provides a uniform grayness over the contacted area.

The literature indicates some inconsistency in the measured values of wringing film thickness. These measurements range from -2.4 to $+2.8 \mu \mathrm{in}$. for lapped metallic surfaces. For optically polished glass surfaces, the recent measurements by Bruce and Thornton [1] ${ }^{1}$ indicate a variation in thickness for different surfaces of $\pm 0.4 \mu \mathrm{in}$. Negative values are explained by the investigators as being caused by an intermeshing of the high points of the surfaces. Although some of this inconsistency in results may be due to systematic error or the use of questionable assumptions, there is a distinct possibility that wringing film thickness is dependent upon the material forming the surface, the planeness of the surfaces involved and the surface finish. However, there is evidence that the film thickness is not critically dependent upon the type of oil used as a wringing agent where this is employed to purposely contaminate lapped surfaces to facilitate contacting [2,3]. Furthermore, whereas different values of wringing film thickness have been reported, many investigators agree that the thickness of a wringing film between two given surfaces is repeatable to a fraction of a microinch $[1,2,3]$.

For the measurement of the practical length of gage blocks to an accuracy of $0.1 \mu \mathrm{in}$., it is obvious that stringent control and measurement of wringing film thickness are required. Practically speaking, it is useless to devise methods of length measurement of gage blocks that eliminate wringing films when the blocks measured will eventually be used in wring combinations with other blocks to achieve specifically desired lengths. What seems to be required is a measurement facility that will directly determine the wringing film thickness between two surfaces to an accuracy better than 0.01 interference fringe $(0.1 \mu \mathrm{in}$.).

Another common occurrence that arises in length measurements is the need to measure the proximity of a plane surface and a spherical surface. This proximity may be either posi-

1 Figures in brackets indicate the literature references at the end of this paper. 
tive or negative, negative in the sense that a contacting of a spherical and plane surface will result in deformation if a contact pressure is present. Although this deformation may be determined theoretically, it is questionable in all cases, particularly in the case of small contact pressure, that the theoretical assumptions are valid. In the case of positive proximity, an interference pattern of Newton's Rings is frequently used to determine the distance between a plane and the vertex of a spherical surface. Figure 1A shows such a pattern formed between a glass surface and a steel ball. The diameter of at least one ring is obtained for each of several wavelengths of light. Knowing the approximate diameter, the distance between the plane and the ball can be determined by extrapolating the fractional order patterns to the vertex and applying the method of coincidences. As in all interference length measurements, correction for phase change at reflection must be made, but in this case correction for dispersion of phase change is also required. Furthermore, the distance between ball and plane must be held constant during the time required to obtain interference patterns for each wavelength.

This paper indicates the results of an investigation into the possibility of employing frustrated total reflection to the problems described above.
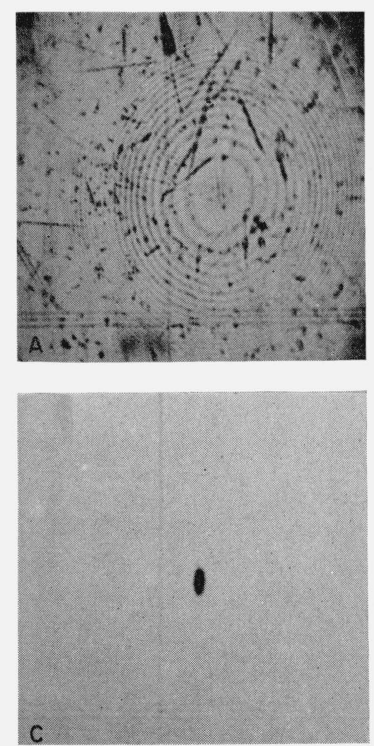
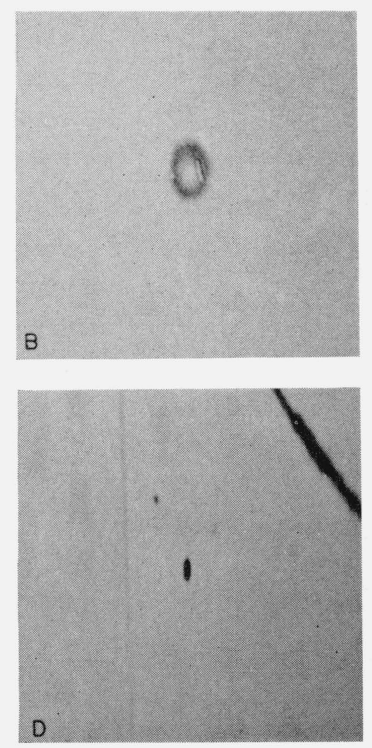

Figure 1A. Newton's Rings obtained by interference between glass boundary surface and steel ball of figure 9 .

Angle of incidence is less than the ${ }^{-}$critical angle. B,C,D. Reflectance patterns obtained by frustrated total reflection at angles 45,65 , and $75^{\circ}$.

\section{Theory}

Total internal reflection occurs when light is incident at an angle $\theta$ upon an interface between two media having indices of refraction $\mu_{1}$ and $\mu_{2}$ such that $\frac{\mu_{1}}{\mu_{2}} \sin \theta>1$ where $\mu_{1}>\mu_{2}$. Here $\mu_{1}$ and $\mu_{2}$ refer to the refractive indices of the incident and refractive media respectively. However, the energy associated with the incident light is transmitted into the refractive medium a short distance before it returns into the incident medium [4].

If a third material is positioned in the region of this energy transmission, this material will transmit or absorb part of the energy and prevent its return to the incident medium. This effect is referred to as frustrated total reflection.

It has been shown that for an all-dielectric system, the relative amount of energy intercepted depends upon the polarization of the incident light, the thickness of the refractive film, the optical constants of the three media involved, the wa velength of the incident light and the angle of incidence [5]. 
In this development of the theory of frustrated total reflection involving metallic frustrating media, it is assumed that a right-hand coordinate system, as shown in figure 2 , is used.

Light from the positive $Z$ space having a refractive index $\mu_{1}$ is incident at an angle $\theta_{1}$ upon an interface represented by the $x y$ plane at $Z=0$. A dielectric film of refractive index $\mu_{2}$ exists between planes $Z=0$ and $Z=-d$. Let $\theta_{2}$ represent the angle of refraction in this medium. A boundary surface exists at $Z=-d$, forming an interface between the dielectric film and a medium having refractive index $\mu_{3}$, where $\mu_{3}$ may be complex. Let $\theta_{3}$ represent the angle of refraction in this medium.

The electric vectors represented in figure 2 are the resultant of all incident, transmitted, and reflected waves. For instance, $E_{1 \mathrm{p}}^{\prime}$ is the resultant of parallel polarizations of all components that are contributed by multireflections from surfaces at $Z=0$ and $Z=-d$.

The components of the electric vectors polarized in the plane of incidence are indicated by $E_{\nu p}$ where $\nu$ is an integer indicating the medium. Prime quantities refer to components associated with reflected waves. Positive components are indicated by the arrows in figure 2. Components of the electric vectors polarized perpendicularly to the plane of incidence are indicated by $E_{\nu s}$. Positive components of $E_{\nu s}$ have a direction parallel to the positive $y$ axis.

In general, the parallel and the perpendicular components of the electric vectors may be expressed by

$$
\begin{array}{ll}
E_{\nu s}=A_{\nu s} e^{i\left[\omega t-\frac{2 \pi}{\lambda_{\nu}}\left(x \sin \theta_{\nu}-Z_{\nu} \cos \theta_{\nu}\right)\right]} & \nu=1,2,3 \\
E_{\nu p}^{\prime}=A_{\nu p} e^{i\left[\omega t-\frac{2 \pi}{\lambda_{\nu}}\left(x \sin \theta_{\nu}-Z_{\nu} \cos \theta_{\nu}\right)\right]} & Z_{1}=Z, \infty \geq Z \geq 0 \\
E_{\nu s}^{\prime}=A_{\nu s}^{\prime} e^{i\left[\omega t-\frac{2 \pi}{\lambda_{\nu}}\left(x \sin \theta_{\nu}+Z_{\nu} \cos \theta_{\nu}\right)\right]} & Z_{2}=Z, 0 \geq Z \geq-d \\
E_{\nu p}^{\prime}=A_{\nu p}^{\prime} e^{i\left[\omega t-\frac{2 \pi}{\lambda_{\nu}}\left(x \sin \theta_{\nu}+Z_{\nu} \cos \theta_{\nu}\right)\right]} & Z_{3}=Z,-d \geq Z \geq-\infty
\end{array}
$$

where

$A$ is the amplitude of the electric vector $\omega$ is the angular velocity $t$ is the time

Figure 2. Representation of the resultant electric vectors of light polarized parallel to the plane of incidence for all incident, reflected, and transmitted waves.

Plane of incidence is in the plane of the paper.

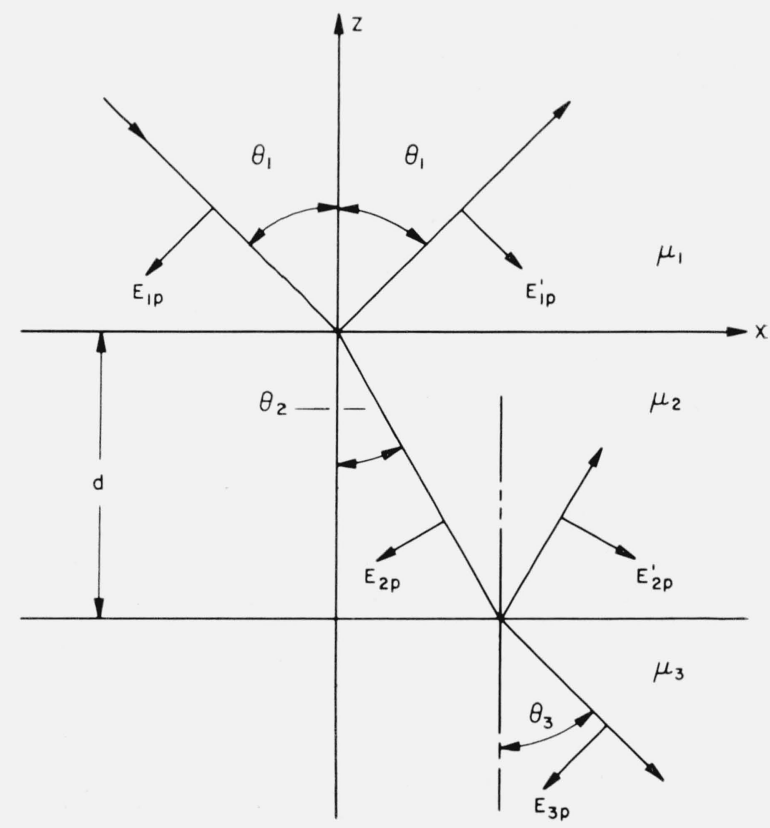


and $\lambda_{\nu}$ is the wavelength in medium $\nu$.

From Snell's Law

where

$$
\begin{aligned}
& \sin \theta_{1}=n_{12} \sin \theta_{2} \\
& \sin \theta_{2}=n_{23} \sin \theta_{3}
\end{aligned}
$$

and

$$
n_{12}=\frac{\mu_{2}}{\mu_{1}}
$$

$$
n_{23}=\frac{\mu_{3}}{\mu_{2}}
$$

Applying the conditions of continuity for electromagnetic waves at the boundaries $Z=0$ and $Z=-d$, the relationships between amplitudes are obtained.

For the polarization perpendicular to the plane of incidence

$$
\begin{aligned}
& A_{1 s}+A_{1 s}^{\prime}=A_{2 s}+A_{2 s}^{\prime} \\
& A_{1 s}-A_{1 s}^{\prime}=\left(A_{2 s}-A_{2 s}^{\prime}\right) n_{12} \frac{\cos \theta_{2}}{\cos \theta_{1}} \\
& A_{2 s} e^{u}+A_{2 s}^{\prime} e^{-u}=A_{3 s} e^{q} \\
& A_{2 s} e^{u}-A_{2 s}^{\prime} e^{-u}=A_{3 s} e^{q} n_{23} \frac{\cos \theta_{3}}{\cos \theta_{2}}
\end{aligned}
$$

and for the polarization parallel to the plane of incidence

$$
\begin{aligned}
& A_{1 p}^{\prime}-A_{1 p}=\left(A_{2 p}^{\prime}-A_{2 p}\right) \frac{\cos \theta_{2}}{\cos \theta_{1}} \\
& A_{1 p}^{\prime}+A_{1 p}=n_{12}\left(A_{2 p}+A_{2 p}^{\prime}\right) \\
& A_{2 p}^{\prime} e^{-u}-A_{2 p} e^{u}=-A_{3 p} e^{q} \frac{\cos \theta_{3}}{\cos \theta_{2}} \\
& A_{2 p}^{\prime} e^{-u}+A_{2 p} e^{u}=n_{23} A_{3 p} e^{q}
\end{aligned}
$$

where

$$
u=\frac{-i 2 \pi \mu_{2} d \cos \theta_{2}}{\lambda_{0}}
$$

and

$$
q=\frac{-i 2 \pi \mu_{3} d \cos \theta_{3}}{\lambda_{0}}
$$

where

$$
\lambda_{0}=\text { vacuum wavelength }
$$

The ratios of the reflected to the incident amplitudes are obtained for the perpendicular and for the parallel polarizations by the use of eqs (3) and (4), respectively.

$$
\frac{-A_{1 s}^{\prime}}{A_{1 s}}=\frac{\cosh u\left(1-\frac{\cos \theta_{1}}{n_{12} n_{23} \cos \theta_{3}}\right)+\sinh u\left(\frac{\cos \theta_{1}}{n_{12} \cos \theta_{2}}-\frac{\cos \theta_{2}}{n_{23} \cos \theta_{3}}\right)}{\cosh u\left(1+\frac{\cos \theta_{1}}{n_{12} n_{23} \cos \theta_{3}}\right)-\sinh u\left(\frac{\cos \theta_{1}}{n_{12} \cos \theta_{2}}+\frac{\cos \theta_{2}}{n_{23} \cos \theta_{3}}\right)}
$$

and

$$
\frac{A_{1 p}^{\prime}}{A_{1 p}}=\frac{\cosh u\left(\frac{\cos \theta_{3}}{n_{12} n_{23} \cos \theta_{2}}-\frac{\cos \theta_{1}}{\cos \theta_{2}}\right)+\sinh u\left(\frac{\cos \theta_{1} \cos \theta_{3}}{n_{23} \cos ^{2} \theta_{2}}-\frac{1}{n_{12}}\right)}{\sinh u\left(\frac{\cos \theta_{1} \cos \theta_{3}}{n_{23} \cos ^{2} \theta_{2}}+\frac{1}{n_{12}}\right)-\cosh u\left(\frac{\cos \theta_{3}}{n_{12} n_{23} \cos \theta_{2}}+\frac{\cos \theta_{1}}{\cos \theta_{2}}\right)} \cdot
$$


For the condition of total internal reflection

and from eq $(2)$

$$
\frac{\sin ^{2} \theta_{1}}{n_{12}^{2}}>1
$$

$$
\cos \theta_{2}=\frac{-i}{n_{12}} \sqrt{\sin ^{2} \theta_{1}-n_{12}^{2}}{ }^{2} .
$$

Using this value of $\cos \theta_{2}$ in eqs (5) and (6), the ratio of reflected to incident amplitudes are

$$
\frac{-A_{1 s}^{\prime}}{A_{1 s}}=\frac{\sinh u\left[\sin ^{2} \theta_{1}-n_{12}^{2}+n_{12} n_{23} \cos \theta_{1} \cos \theta_{3}\right]+i \sqrt{\sin ^{2} \theta_{1}-n_{12}^{2}}\left[\cos \theta_{1}-n_{12} n_{23} \cos \theta_{3}\right] \cosh u}{\sinh u\left[\sin ^{2} \theta_{1}-n_{12}^{2}-n_{12} n_{23} \cos \theta_{1} \cos \theta_{3}\right]-i \sqrt{\sin ^{2} \theta_{1}-n_{12}^{2}}\left[\cos \theta_{1}+n_{12} n_{23} \cos \theta_{3}\right] \cosh u}
$$

for the perpendicular polarization, and

$$
\frac{A_{1 p}^{\prime}}{A_{1 p}}=\frac{\sinh u\left[n_{12}^{3} \cos \theta_{1} \cos \theta_{3}+n_{23}\left(\sin ^{2} \theta_{1}-n_{12}^{2}\right)\right]-i \sqrt{\sin ^{2} \theta_{1}-n_{12}^{2}}\left[n_{12} \cos \theta_{3}-n_{12}^{2} n_{23} \cos \theta_{1}\right] \cosh u}{\sinh u\left[n_{12}^{3} \cos \theta_{1} \cos \theta_{3}-n_{23}\left(\sin ^{2} \theta_{1}-n_{12}^{2}\right)\right]+i \sqrt{\sin ^{2} \theta_{1}-n_{12}^{2}}\left[n_{12} \cos \theta_{3}+n_{12}^{2} n_{23} \cos \theta_{1}\right] \cosh u}
$$

for the parallel polarization.

Equations (8) and (9) may be used to determine the magnitude and phase of the reflected electric vector when an all-dielectric system is being considered. For such a system the reflectance for the perpendicular component is given by

where

$$
R_{s}=\frac{A_{1 s}^{\prime} A_{1 s}^{\prime *}}{A_{1 s}^{2}}=\frac{B-C}{B+C}
$$

$$
\begin{aligned}
& B=n_{12}^{2}\left(1-n_{23}^{2}\right)\left(n_{12}^{2}-1\right) \sinh ^{2} u-\left(\sin ^{2} \theta_{1}-n_{12}^{2}\right)\left[2 \sin ^{2} \theta_{1}-n_{12}^{2} n_{23}^{2}-1\right] \\
& C=2\left(\sin ^{2} \theta_{1}-n_{12}^{2}\right) \cos \theta_{1} \sqrt{n_{12}^{2} n_{23}^{2}-\sin ^{2} \theta_{1}}
\end{aligned}
$$

and the reflectance for the parallel component is given by

where

$$
R_{p}=\frac{A_{1 p}^{\prime} A_{1 p}^{*}}{A_{1 p}^{2}}=\frac{D+E}{D-E}
$$

$$
\begin{aligned}
D=\frac{\left(\sin ^{2} \theta_{1}-n_{12}^{2}\right)}{n_{23}^{2}}\left[n_{12}^{2} n_{23}^{2}\left(1+n_{12}^{2} n_{23}^{2}\right)-\sin ^{2} \theta_{1}\left(1+n_{12}^{4} n_{23}^{4}\right)\right] \\
\quad+\frac{\sinh ^{2} u}{n_{23}^{2}}\left[\sin ^{2} \theta_{1}\left(1-n_{12}^{4}\right)+n_{12}^{2}\left(n_{12}^{2}-1\right)\right]\left[\left(n_{23}^{4}-1\right) \sin ^{2} \theta_{1}+n_{12}^{2} n_{23}^{2}\left(1-n_{23}^{2}\right)\right]
\end{aligned}
$$

$E=-2 n_{12}^{2} \cos \theta_{1}\left(\sin ^{2} \theta_{1}-n_{12}^{2}\right) \sqrt{n_{12}^{2} n_{23}^{2}-\sin ^{2} \theta_{1}}$.

When the frustrating medium has a complex refractive index, i.e., when $\mu_{3}=n(1-i \alpha)$ where $n$ is the refractive index and $\alpha$ is the absorption index, $n_{23}$ and $\cos \theta_{3}$ become complex and eqs (8) and (9) must be modified. From eq (2),

and

$$
\cos \theta_{3}=\frac{-1}{n_{12} n_{23}}{\sqrt{n_{12}^{2} n_{23}^{2}-\sin ^{2} \theta_{1}}}^{3}
$$

$$
n_{23}=\frac{n(1-i \alpha)}{\mu_{2}}
$$

\footnotetext{
2 The sign of $\cos \theta_{2}$ is chosen negative as this results in a dampening of the electric vector $E_{2}$ as it progresses in the film medium in a negative $z$ direction.

3 The sign of $\cos \theta_{3}$ is chosen negative as this results in a dampening of the electric vector $E_{3}$ as it progresses in the frustrating medium in a negative $z$ direction.
}

$670682-63-3$ 
Applying these expressions to eqs (8) and (9), one obtains for the perpendicular polarization,

$$
\frac{-A_{1 s}^{\prime}}{A_{1 s}}=\frac{\left[\begin{array}{l}
\sinh u\left[\sin ^{2} \theta_{1}-n_{12}^{2}-\rho^{1 / 2} \cos \theta_{1} \cos \frac{\phi}{2}\right]-\rho^{1 / 2}\left(\sin ^{2} \theta_{1}-n_{12}^{2}\right)^{1 / 2} \sin \frac{\phi}{2} \cosh u \\
-i\left[\rho^{1 / 2} \cos \theta_{1} \sin \frac{\phi}{2} \sinh u-\left(\sin ^{2} \theta_{1}-n_{12}^{2}\right)^{1 / 2}\left(\cos \theta_{1}+\rho^{1 / 2} \cos \frac{\phi}{2}\right) \cosh u\right]
\end{array}\right]}{\left[\begin{array}{l}
\sinh u\left[\sin ^{2} \theta_{1}-n_{12}^{2}+\rho^{1 / 2} \cos \theta_{1} \cos \frac{\phi}{2}\right]-\rho^{1 / 2}\left(\sin ^{2} \theta_{1}-n_{12}^{2}\right)^{1 / 2} \sin \frac{\phi}{2} \cosh u \\
+i\left[\rho^{1 / 2} \cos \theta_{1} \sin \frac{\phi}{2} \sinh u-\left(\sin ^{2} \theta_{1}-n_{12}^{2}\right)^{1 / 2}\left(\cos \theta_{1}-\rho^{1 / 2} \cos \frac{\phi}{2}\right) \cosh u\right]
\end{array}\right]}
$$

and for the parallel polarization,

where,

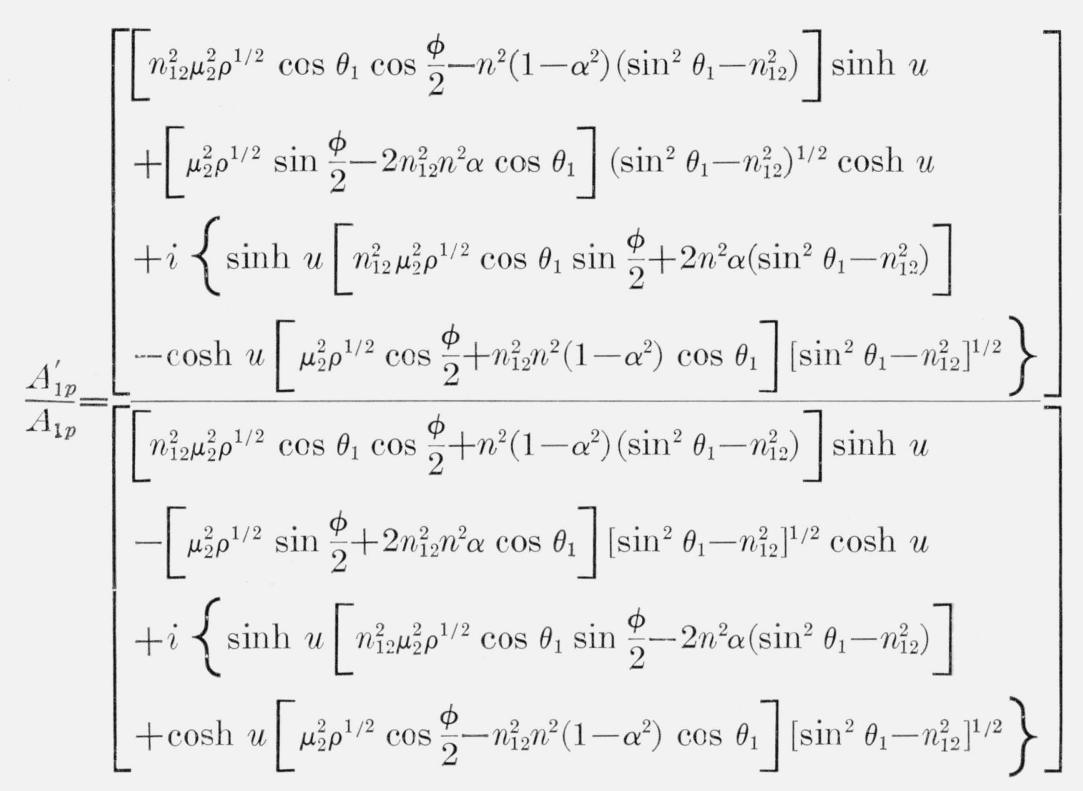

$$
\begin{aligned}
\rho & =+\sqrt{\left[\frac{n_{12}^{2} n^{2}\left(1-\alpha^{2}\right)}{\mu_{2}^{2}}-\sin ^{2} \theta_{1}\right]^{2}+\frac{4 n_{12}^{4} n^{4} \alpha^{2}}{\mu_{2}^{4}}} \\
\sin \phi & =-\frac{2 n_{12}^{2} n^{2} \alpha}{\mu_{2}^{2} \rho} \\
\cos \phi & =\frac{n_{12}^{2} n^{2}\left(1-\alpha^{2}\right)-\mu_{2}^{2} \sin \theta_{1}}{\mu_{2}^{2} \rho} .
\end{aligned}
$$

Equations (12) and (13) may be used to determine the magnitude and phase of the reflected electric vector when the frustrating medium has a complex refractive index, e.g., a metallic material. Reflectance equations similar to eqs (10) and (11), but for the case where $\mu_{3}$ is complex are given by eqs (14) and (15).

$$
\begin{aligned}
R_{s} & =\frac{A_{1 s}^{\prime} A_{1 s}^{\prime *}}{A_{1 s}^{2}} \\
R_{p} & =\frac{A_{1 p}^{\prime} A_{1 p}^{\prime *}}{A_{1 p}^{2}} .
\end{aligned}
$$

Numerical calculations of reflectance can be obtained by evaluating eqs (12) and (13) and multiplying by the numerical complex conjugate. 
For an all-dielectric system where $\mu_{1}=\mu_{3}=1.72 ; \mu_{2}=1.38 ; \theta=60^{\circ}$; the reflectance relationship to film thickness as calculated from eqs (10) and (11) is identical to that calculated by Vasicek [6]. The solid curves of figure 3 are also calculated from eq (10). They show the theoretical relationships of reflectance to film thickness of air between the surfaces of glass having a refractive index of 1.5. The abscissa indicates the film thickness in microinches and the ordinate represents the percentage of perpendicularly polarized mercury green light reflected. Curves for parallel-polarized light, calculated from eq (11), have the same form but exhibit somewhat better sensitivity at larger angles of incidence. For instance, as the film increases from 0 to $0.2 \mu \mathrm{in}$. the intensity of the reflected light incident at $80^{\circ}$ increases from 0 to 10 percent.

The broken curve of figure 3 results from calculation of eq (14). The curve shows the tneoretical relationship between reflectance of perpendicular polarized light and a film thickness of air existing between a "totally reflecting" glass surface and a steel surface. Curves for angles of incidence less than $80^{\circ}$ intercept the ordinate axis at lower values of reflectance but do not change their slope to any significant degree.

Curves derived from calculations of eq (15) are much more interesting. Figure 4 shows, for different angles of incidence, theoretical relationships between reflectance of parallel polarized light and a film thickness of air existing between a "totally reflecting" glass surface and a steel surface. Of significance, is the formation of a minimum occurring at a film thickness dependent upon the angle of incidence. Furthermore, there is indicated a sensitivity in the reflectance to film thickness relationship that essentially equals the sensitivity obtained in the dielectric case. Unlike the dielectric case, a considerable increase in reflectance is obtained as the film thickness approaches zero. The form of the curves shows theoretically that a measurement of reflectance to 1 percent could indicate the thickness of a very thin film of air to an accuracy of 20 billionths of an inch.

From the form of the curves in figure 4 it is apparent that for a very thin film of varying thickness a pattern of minimum reflectance should be obtained resembling an interference fringe of equal thickness. Unlike an interference fringe pattern, only one minimum should be obtained, corresponding to a thickness of film dependent upon the angle of incidence. Therefore no ambiguity concerning the order of interference should exist. Another important difference arises from the sharpness of the reflectance dip obtained for larger angles of incidence. For instance, the half width of a conventional two-beam interference fringe corresponds to approximately 5 millionths of an inch variation in film thickness whereas the half width of these dips can be less than 1 millionth of an inch. ${ }^{4}$

\footnotetext{
${ }_{4}^{4}$ Defining the half width of an unsymmetrical dip is somewhat arbitrary. In this instance the half width is defined as the width of the dip at that reflectance equal to the minimum reflectance plus one-hall the difference is reflectance between the minimum value and the value at
} zero film thickness.

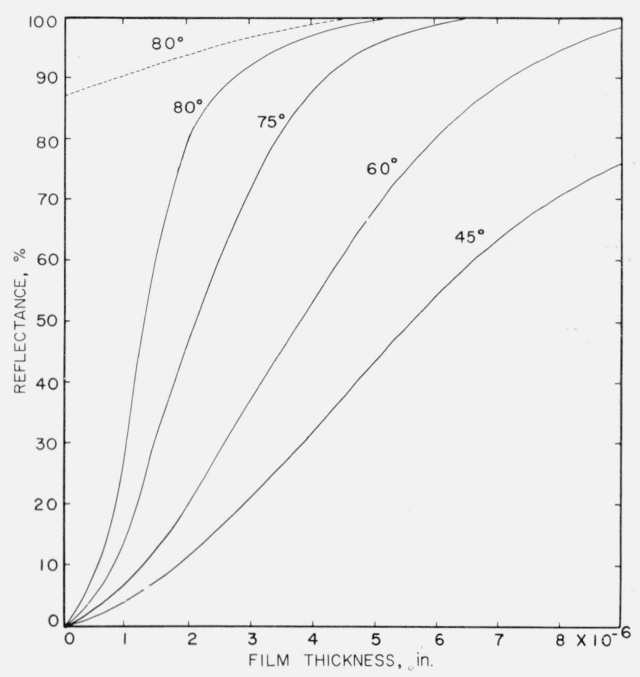

FiguRE 3. Variation of reflectance with film thickness of air at various angles of incidence of perpendicular polarized light.

Solid lines are calculated from eq (10) for an all dielectric case The dotted line obtained from eq (14) is for the case where $\mu_{3}$ is a steel surface of complex index. 


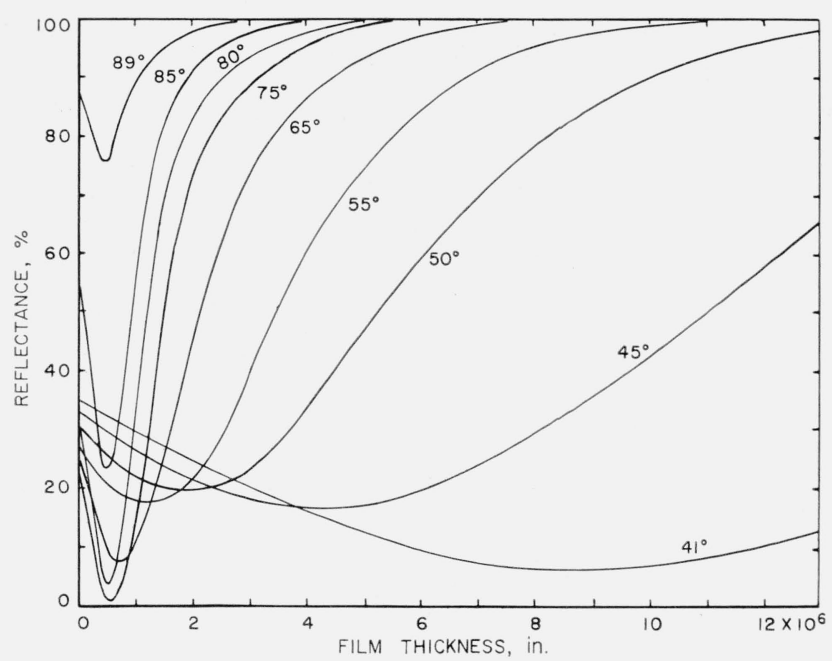

Figure 4. Variation of reflectance with film thickness of air at various angles of incidence of parallel polarized light for the case where $\mu_{3}$ is of complex index.

The curves are calculated from eq (15). Note the unique minima obtained in the reflectance.

The film thicknesses at which the minima are obtained can be calculated from the following equation.

$$
\begin{aligned}
d=\frac{-}{4 \pi \mu_{1}\left(\sin ^{2} \theta_{1}-n_{12}^{2}\right)^{1 / 2}} \cdot \ln \\
\left.\qquad \frac{(A E-B D) \pm \sqrt{(B D-A E)^{2}-[F(A+B)-C(D+E)][F(B-A)+C(D-E)]}}{F(A+B)-C(D+E)}\right]
\end{aligned}
$$

where

where

$$
\begin{aligned}
& A=(a-b)^{2}+(c-\delta)^{2}+(e+f)^{2}+(g+h)^{2} \\
& B=2[(a-b)(c-\delta)-(e+f)(g+h)] \\
& C=(c-\delta)^{2}+(g+h)^{2}-(a-b)^{2}-(e+f)^{2} \\
& D=(a+b)^{2}+(c+\delta)^{2}+(e-f)^{2}+(g-h)^{2} \\
& E=2[(e-f)(g-h)-(a+b)(c+\delta)] \\
& F=(c+\delta)^{2}+(g-h)^{2}-(a+b)^{2}-(e-f)^{2}
\end{aligned}
$$

$$
\begin{aligned}
& a=n_{12}^{2} \mu_{2}^{2} \rho^{1 / 2} \cos \frac{\phi}{2} \cos \theta_{1} \\
& b=n^{2}\left(1-\alpha^{2}\right)\left(\sin ^{2} \theta_{1}-n_{12}^{2}\right) \\
& c=\mu_{2}^{2} \rho^{1 / 2} \sin \frac{\phi}{2}\left(\sin ^{2} \theta_{1}-n_{12}^{2}\right)^{1 / 2} \\
& \delta=2 n_{12}^{2} n^{2} \alpha \cos \theta_{1}\left(\sin ^{2} \theta_{1}-n_{12}^{2}\right)^{1 / 2} \\
& e=n_{12}^{2} \mu_{2}^{2} \rho^{1 / 2} \sin \frac{\phi}{2} \cos \theta_{1} \\
& f=2 n^{2} \alpha\left(\sin ^{2} \theta_{1}-n_{12}^{2}\right) \\
& g=\mu_{2}^{2} \rho^{1 / 2} \cos \frac{\phi}{2}\left(\sin ^{2} \theta_{1}-n_{12}^{2}\right)^{1 / 2} \\
& h=n_{12}^{2} n^{2}\left(1-\alpha^{2}\right) \cos \theta_{1}\left(\sin ^{2} \theta_{1}-n_{12}^{2}\right)^{1 / 2} .
\end{aligned}
$$


Equation 16 is derived by differentiating eq (15) with respect to the function $u$. Setting the differential to zero, solving for $u$ and applying eq (7) to the expression for $u$ in eq (4), one obtains eq (16).

By varying the constants $\mu_{1}, \mu_{2}, \mu_{3}, \lambda$ in eq (13) the location of the minimum is changed as is indicated in figures $5,6,7,8 .{ }^{5}$ Variation of $\mu_{3}$ and $\lambda$ does not significantly change the location of the minimum and no major increase in sensitivity is noticed. However, with an increase in the optical constant, $\mu_{1}$, or the ratio $\frac{\mu_{1}}{\mu_{2}}$, the minimum forms at smaller values of $d$ and the sensitivity significantly increases as there is a larger change in reflectance with changes in $d$.

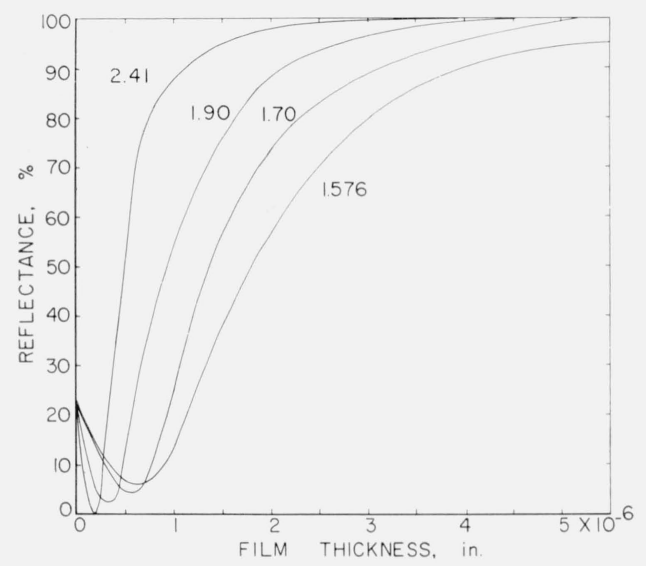

Figure 5. Variation of reflectance with film thickness of air for increasing values of index of $\mu_{1}$ as calculated from eq (15).

[ The angle of incidence of parallel polarized light is $65^{\circ}$.

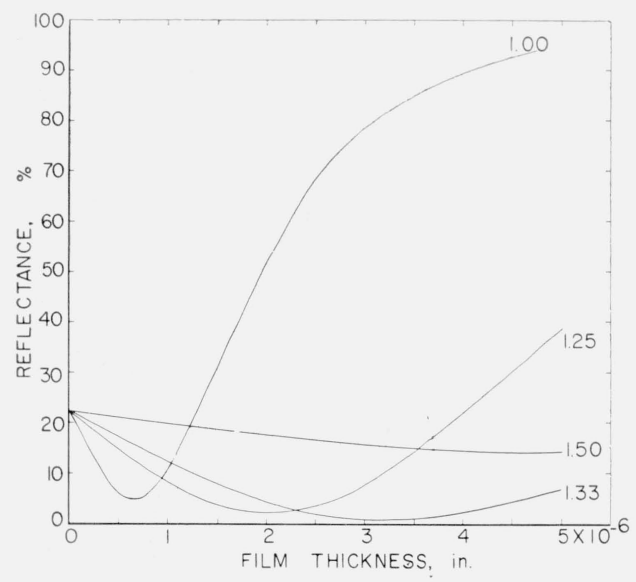

FIGURE 6. Variation of reflectance with film thickness for increasing value of index of the film for parallel polarized light.

The angle of incidence is $65^{\circ}$.

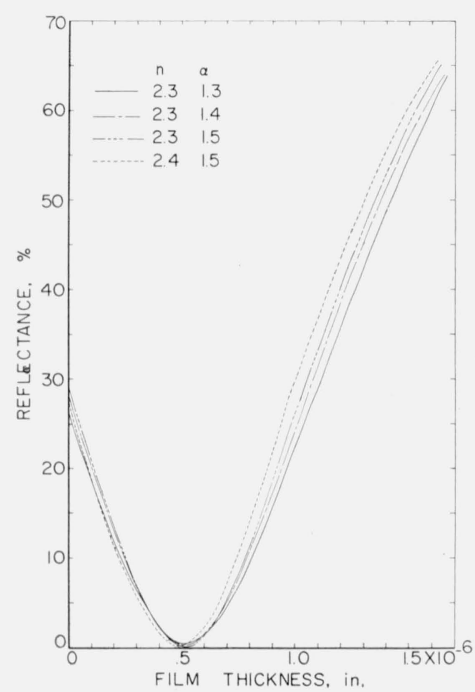

FIGURE 7. Variation of reflectance with film thickness of air for various values of index of $\mu_{3}$.

The angle of incidence is $65^{\circ}$.

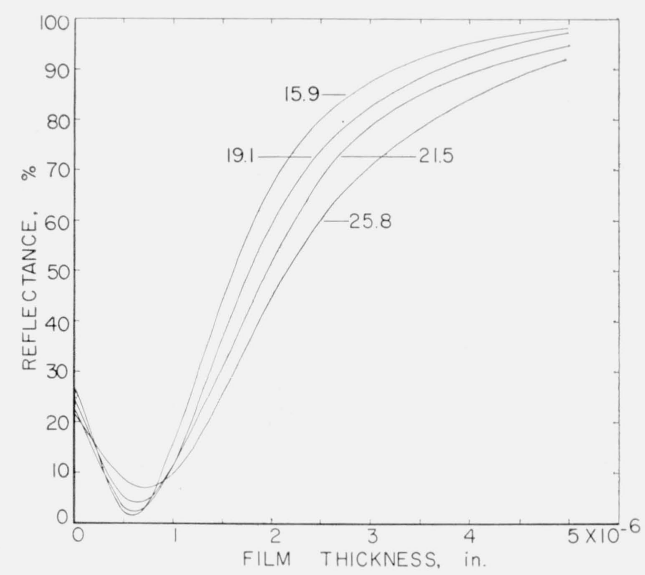

Figure 8. Variation of reflectance with film thickness of air for various values of wavelength given in microinches.

The angle of incidence is $65^{\circ}$.

8 In calculations for varying wavelength, $\mu_{1}$, and $\mu_{3}$ were changed to account for dispersion of glass and steel. 


\section{Experimental Procedure}

To experimentally verify the theoretical eqs (14), (15), and (16) the arrangement drawn in plane view in figure 9 was employed. A variable prism, consisting of a half cylinder of glass with two planoconcave cylindrical lenses of the same refractive index $\left(\mu_{e}=1.576\right)$ contacted to the surface with glycerine, was positioned on a lapped steel table of a spectrometer. The spectrometer rested on a table surface whose normal could be inclined through small angles from the vertical. The autocollimating telescope and the divided circle of the spectrometer were employed to aline each of the planoconcave cylindrical lenses so that the plane surfaces of the lenses formed included angles with the reflecting surface of the prism equal to the desired angle of incidence, $\theta$. The prism assembly was then rotated so that the entrance window of the prism assembly was normal to a beam of plane polarized, collimated white light filtered with a mercury-green interference filter. Using the tilt table supporting the spectrometer, the normal to the spectrometer table was inclined one degree from the vertical in such a direction as to fall in the plane defined by the vertical axis and the normal to the reflecting surface of the prism. A lapped S.A.E. 52100 steel ball of 1 in. diam and having a mass known to 1 percent was positioned on the spectrometer table. This platform served as an inclined plane holding the ball against the reflecting surface of the prism with a computed contact load of $0.1 \mathrm{~g}$.

The telescope of the spectrometer was replaced with a calibrated microscope having a 32 mm objective and a $10 \mathrm{X}$ filar eyepiece. The microscope was positioned to receive the reflected beam and was focused on the totally reflecting surface of the prism at the point where the ball made contact.

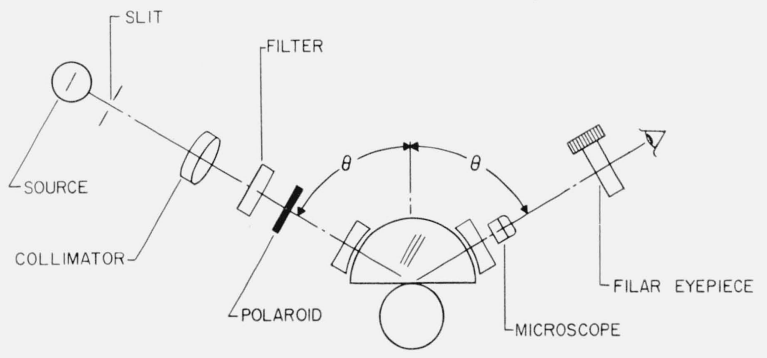

FIGURE 9. Schematic outline of setup used in experimental verification of theory.

The basic equipment consists of a spectrometer with the vertical axis of rotation being perpendicular to the plane of the paper. The prism, end caps, and ball rests on the spectrometer table with the ball held against the prism by a $0.1 \mathrm{~g}$ force due to a $1 \mathrm{deg}$ tilt in the table.

\section{Results}

With the incident light perpendicularly polarized a slight diminishing in intensity of the reflected beam at and surrounding the point of contact was observed. This qualitatively verified the result indicated by eq (14) (see the broken line curve of figure 3). No minima were observed for this condition.

With the incident light parallel polarized, minima such as those predicted by eq (15) were obtained. Figure 1, B, C, D illustrates typical patterns observed at different angles of incidence larger than the critical angle. These elliptical patterns, having major diameters as small as 0.0015 in., were all obtained at the same magnification as that used for the Newton Ring pattern shown in figure $1 \mathrm{~A}$. The film thickness at which the minimum occurs is proportional to the second power of the diameter as measured along the major axis of the elliptical ring. The existence of a unique mimimum rather than the cyclic pattern shown in figure $1 \mathrm{~A}$, and the effect of increasing angle of incidence upon ring size, sharpness, and contrast qualitatively verify eq (15) as represented by the calculated curves shown in figure 4. The irregularities of the ring pattern for $45^{\circ}$, shown in figure $1 \mathrm{~B}$, are considered to be the effect of surface irregularity of the steel ball. This same surface finish appears to have less effect on the minima obtained at larger angles of incidence. 
A quantitative verification of eqs (15) and (16) was obtained by comparing the experimental determinations of film thickness at which the minima are formed with results derived from calculations of eq (16). To experimentally determine the film thickness $x$, the major diameters, $2 y$, of the elliptical rings formed at known angles of incidence were measured with the calibrated filar eyepiece of the microscope. These measured values were applied to the sagittal equation

$$
x=R-\left(R^{2}-y^{2}\right)^{1 / 2},
$$

where $R$ is the radius of the steel ball.

The experimental results are compared in table 1 with the theoretical results as determined by eq (16). The experimental results and the estimated error associated with the particular measurement indicate clearly the close agreement of experiment with theory. In every case except one the theory falls within the experimental error. The amount of error indicates primarily the ability of the observer to set a crosshair on the minimum ring. With increasing angle of incidence the pattern becomes sharper and therefore easier to set on.

TABle 1. Comparison between experimental and theoretical determinations of film thickness

\begin{tabular}{c|c|c|c}
\hline \hline$\theta_{1}$ & Exp. values & Theor. values & Diff. \\
Degrees & Microinches & Microinches & Microinches \\
41 & $6.6 \pm 1.2$ & 7.0 & 0.4 \\
45 & $2.58 \pm .24$ & 2.60 & .02 \\
55 & $1.13 \pm .15$ & .98 & .15 \\
65 & $.47 \pm .12$ & .63 & .16 \\
75 & $.49 \pm .09$ & .49 & .00 \\
\hline
\end{tabular}

In the theoretical determination no factor was used to compensate for the surface finish of the ball. However, agreement between theoretical and experimental values was essentially $0.1 \mu \mathrm{in}$. The predominant scratch depth of the ball was approximately $2 \mu \mathrm{in}$. This indicates the phenomena are practically independent of surface finish.

\section{Conclusions}

Preliminary investigation and results indicate the method of frustrated total reflection applicable to the precise measurement of thin films such as those existing between a dielectric and conductor. Significant in this investigation are that the measurements are independent of any phase change due to surface finish and the minimum obtained is single valued, eliminating the necessity of establishing an order as with fringes.

\section{References}

[1] C. F. Bruce and B. S. Thornton, J. Appl. Phys. 2\%, 853 (1956).

[2] A. M. Dexter, ASTE 59, Tech. Paper 195 (1959).

[3] F. H. Rolt and H. Barrell, Proc. Royal Soc. A116, 401 (1927).

[4] R. W. Ditchburn, Light, Interscience Publishers, Inc., N.Y., 434 (1955).

[5] E. E. Hall, Phys. Rev. 15, 73 (1902).

[6] A. Vasicek, Optics of Thin Films, Trans. by Mrs. H. Watney-Kaczer, North Holland Pub. Company Amsterdam. Interscience Publishers, New York (1960).

(Paper 67A2-199) 